\section{Transtornos mentais comuns e qualidade de vida em jovens: uma amostra populacional de Pelotas, Rio Grande do Sul, Brasil}

\author{
Mental common disorders and quality of life \\ in young adulthoods: a population-based sample \\ in Pelotas, Rio Grande do Sul State, Brazil
}

\author{
1 Universidade Católica de \\ Pelotas, Pelotas, Brasil. \\ Correspondência \\ T. C. Mondin \\ Universidade Católica de \\ Pelotas. \\ Rua Dr. Fernando Ferrari 257, \\ apto. 302, Pelotas, RS \\ 96080-090, Brasil. \\ thaisemondin@yahoo.com.br
}

\begin{abstract}
This study aimed to assess the prevalence of common mental disorders (CMD) and their association with quality of life among young adults in Pelotas, Rio Grande do Sul State, Brazil. This was a cross-sectional population-based study of young people 18 to 24 years of age. Sampling used simple cluster selection. Investigation of CMD used the Self-Report Questionnaire (SRQ-20), while quality of life was measured with the Medical Outcomes Survey Short-Form General Health Survey (SF-36). Prevalence of CMD in the study sample was $24.5 \%(N=382)$, and was positively associated with: female gender, lower socioeconomic status (classes D or E), not studying, not working, alcohol consumption, smoking, and substance abuse. Young adults with CMD had lower mean scores on the SF-36 in all the assessed quality of life domains. Measures to prevent CMD are necessary to provide better quality of life for this age group.
\end{abstract}

Mental Disorders; Mental Health; Quality of Life

\author{
Karen Jansen 1 \\ Thaíse Campos Mondin 1 \\ Liliane da Costa Ores 1 \\ Luciano Dias de Mattos Souza 1 \\ Caroline Elizabeth Konradt 1 \\ Ricardo Tavares Pinheiro 1 \\ Ricardo Azevedo da Silva 1
}

\section{Introdução}

A adolescência e a vida adulta jovem caracterizam-se por mudanças físicas, psíquicas e sociais. Nessa fase há uma predisposição ao desenvolvimento de alguns transtornos psicopatológicos como a depressão, ansiedade e alguns comportamentos de risco à saúde como uso de drogas e álcool 1. Estudos populacionais no Brasil demonstram uma prevalência de transtornos mentais comuns (TMC) que variam de $17 \%$ a $35 \% 2,3,4,5$. Em estudos internacionais a prevalência varia de $15,4 \%$ a $30,5 \% 6,7,8,9$.

Transtornos mentais comuns foram conceituados por Goldberg \& Huxley 10, incluindo depressão não-psicótica, ansiedade e sintomas somatoformes. Os TMC abrangem sintomas como: insônia, fadiga, esquecimento, irritabilidade, dificuldades de concentração, queixas somáticas e sentimento de inutilidade 10 . Transtornos mentais comuns são comumente encontrados em indivíduos com baixa classe socioeconômica, mulheres e separados 11. Usuários de tabaco e álcool, assim como comportamento sedentário também mostraram associações com TMC 2 . Estudos encontraram relações de TMC com vulnerabilidade social tais como baixa escolaridade, menor número de bens, condições precárias de moradia, baixa renda e desemprego 12 .

O conceito de qualidade de vida atraiu o público científico na última década para pesquisas que se relacionam a variáveis sociodemográficas 
e culturais, além de doenças somáticas ${ }^{13}$. Pesquisas da saúde mental centram-se em questões como a disfunção, incapacidade e deficiência que a baixa qualidade de vida pode ocasionar. Indivíduos com transtornos de ansiedade e depressão têm o estado de saúde bastante comprometido quando comparados a indivíduos sem esses transtornos 14,15.

Qualidade de vida segundo a Organização Mundial da Saúde (OMS) 16 "é um conceito de alcance abrangente, afetado de forma complexa por sua saúde física, estado psicológico e nível de independência, por suas relações sociais e relações com as características do seu meio ambiente" (p. 1403). Há maior preocupação em avaliar a qualidade de vida da população geral, especialmente nas últimas duas décadas 17 .

São amplamente reconhecidas e estudadas as questões referentes à saúde mental e qualidade de vida, uma vez que são fundamentais para uma organização mais efetiva do sistema de saúde e o planejamento de promoção em saúde. Em uma revisão bibliográfica sobre TMC foi possível verificar um aumento da ocorrência de TMC na atenção básica e nas comunidades 18. Além disso, a ocorrência de TMC pode refletir na baixa qualidade de vida dos jovens, justificando então a necessidade de estudos que avaliem tal relação, já que a percepção de saúde geral do indivíduo está diretamente relacionada ao bem-estar físico e psicológico. Assim, o objetivo deste estudo foi verificar a prevalência de TMC e sua associação com qualidade de vida em jovens de 18 a 24 anos da cidade de Pelotas, Rio Grande do Sul, Brasil.

\section{Método}

Estudo transversal de base populacional com jovens de 18 a 24 anos, residentes da zona urbana da cidade de Pelotas. A seleção amostral dos sujeitos foi realizada por conglomerados, considerando a população de 39.667 jovens entre 18 e 24 anos e a divisão censitária atual de 448 setores na cidade, fornecida pelo Instituto Brasileiro de Geografia e Estatística (IBGE; http://www.ibge.gov.br).

A fim de garantir a inclusão da amostra necessária, 89 setores censitários foram sorteados sistematicamente. A seleção dos domicílios nos setores sorteados foi realizada segundo uma amostragem sistemática, sendo o primeiro domicílio a residência da esquina pré-estabelecida pelo IBGE como início do setor, o intervalo de seleção foi determinado por um pulo de dois domicílios entre os sorteados.

Após a identificação dos jovens, alunos do Centro de Ciência da Vida e da Saúde da Universidade Católica de Pelotas, devidamente treina- dos, explicaram os objetivos e os instrumentos do estudo. Foram incluídos no estudo, os jovens sorteados que aprovaram a participação e assinaram o Termo de Consentimento Livre e Esclarecido e a seguir participaram de uma entrevista que coletou dados sociodemográficos, qualidade de vida e TMC, além disso, responderam a um questionário autoaplicável sobre abuso de substâncias psicoativas. Foram excluídos os jovens que por algum motivo físico ou cognitivo não foram capazes de compreender os objetivos do estudo ou assinar o termo de consentimento, bem como aqueles que não sabiam ler ou escrever.

A coleta de dados foi realizada por intermédio de questionário constituído pelas seguintes variáveis: idade, sexo, classificação socioeconômica, estudo, trabalho, uso de substâncias psicoativas na última semana e nos últimos três meses. A avaliação socioeconômica dos participantes foi realizada por Critério de Classificação Econômica da Associação Brasileira de Empresas de Pesquisa (ABEP), que se baseia no acúmulo de bens materiais e na escolaridade do chefe da família. Esses critérios enquadram as pessoas em classes (A, B, C, D, ou E) com base em escores alcançados. A letra "A" refere-se à classificação socioeconômica mais alta e "E" à mais baixa (Associação Brasileira de Empresas de Pesquisa. Critério de classificação econômica Brasil. http://www. abep.org/novo/Content.aspx?ContentID=301, acessado em Mai/2008).

Para a investigação dos TMC foi aplicado o Self-Report Questionnaire (SRQ-20). Composto por 20 questões sobre sintomas psicossomáticos para rastreamento de transtornos não psicóticos, incluindo sintomas depressivos, ansiosos e queixas somáticas. O SRQ-20 é recomendado pela OMS para estudos comunitários e em atenção básica à saúde 19 . O ponto de corte utilizado foi 7, que de acordo com a validação de Gonçalves et al. 20 apresenta os seguintes padrões psicométricos: sensibilidade de $86,33 \%$ e especificidade de $89,31 \%$.

Os níveis de qualidade de vida foram mensurados pela Medical Outcomes Survey Shortform General Health Survey (SF-36), escala que consiste em 36 itens que abordam limitações na vida diária em razão de problemas de saúde, proporcionando uma estimativa subjetiva do estado funcional do indivíduo. A SF-36 resulta em uma classificação de oito domínios: capacidade funcional, aspecto físico, dor, vitalidade, aspecto social, aspecto emocional, saúde mental e estado geral de saúde; avaliados mediante a transformação das respostas em escores escala de 0 a 100, em que quanto maior a pontuação melhor a qualidade de vida. Os coeficientes de confiabilidade para os oito domínios da SF-36 oscilaram de 0,77 
a 0,94 , com um valor médio de 0,82 , e a validade variou entre 0,51 e 0,85 21 .

Após a codificação dos instrumentos, o processamento dos dados foi realizado com dupla entrada no programa Epi Info 6.04d (Centers for Disease Control and Prevention, Atlanta, Estados Unidos) com checagem automática no momento da digitação. Além disso, foi testada no mesmo programa a consistências entre as duas entradas de dados. Para o tratamento estatístico, foi utilizado o programa Stata 9.0 (Stata Corp., College Station, Estados Unidos) no qual se procedeu à análise inicial com objetivo de obter a frequência de TMC, além de caracterizar a amostra do estudo. O teste estatístico qui-quadrado foi utilizado na análise bivariada dos dados, visando à descrição de associações entre a ocorrência TMC e as variáveis independentes investigadas. Para a comparação entre as médias dos oito domínios de qualidade de vida e variáveis independentes, foi realizado o teste $t$ de Student.

A análise multivariada foi realizada por regressão linear em que as variáveis com valor de $\mathrm{p}<0,2$ na análise bivariada foram incluídas em um modelo teórico de análise cujas variáveis independentes foram divididas em três níveis hierárquicos, levando em consideração os fatores que supostamente podem confundir a associação entre TMC e qualidade de vida 22 . O primeiro nível incluiu as variáveis sociodemográficas (sexo, idade, classificação socioeconômica, estudo e trabalho), enquanto o segundo nível foi composto por substâncias psicoativas, restando ao terceiro nível do modelo a presença de TMC. Nesse modelo de análise, as variáveis de nível abaixo foram controladas para as do nível antecessor, ou seja, após o ajuste entre as variáveis de mesmo nível hierárquico, aquelas com valor de $\mathrm{p}<0,2$ foram incluídas na análise do nível abaixo. Após a análise multivariada, foram consideradas estatisticamente significativas as associações com valor de $\mathrm{p} \leq 0,05$.

\section{Resultados}

Neste estudo transversal de base populacional, foram identificados 1.762 jovens de 18 a 24 anos entre os 89 setores censitários incluídos, porém $202(11,5 \%)$ jovens recusaram-se a participar do estudo. Assim, a amostra final foi composta por 1.560 jovens de 18 a 24 anos de idade. Desses, foram $56,4 \%$ do sexo feminino, $48,1 \%$ pertenciam à classificação socioeconômica $\mathrm{C}$ de acordo com a Associação Brasileira de Empresas de Pesquisa (ABEP), 46\% estavam estudando no momento da entrevista e 59,6\% estavam trabalhando. Quanto ao uso de substância psicoativas, 26,9\% consu- miram álcool pelo menos uma vez por semana, 24,9\% usaram tabaco no mínimo uma vez por semana, 7,4\% utilizaram alguma outra substância psicoativa nos últimos três meses.

A prevalência de TMC na amostra estudada foi de $24,5 \%(n=382)$. As associações significativas na análise bivariada foram: ser do sexo feminino $(\mathrm{p}<0,001)$, pertencer à classe socioeconômica D ou $\mathrm{E}(\mathrm{p}<0,001)$, não estar estudando ( $\mathrm{p}<0,004)$, não estar trabalhando atualmente ( $\mathrm{p}<0,001)$, consumir álcool pelo menos uma vez na semana $(\mathrm{p}=0,008)$. A idade foi a única variável que não se mostrou associada com TMC ( $\mathrm{p}=0,306$ ) (Tabela 1).

Na Tabela 2, pode-se observar a associação entre as variáveis independentes investigadas em relação aos domínios de qualidade de vida, na qual aquelas com $\mathrm{p}<0,2$ e que também estiveram associadas à ocorrência de TMC, foram consideradas possíveis fatores de confusão.

Todos os domínios do SF-36 (capacidade funcional, aspectos físicos, dor, estado geral de saúde, vitalidade, aspectos sociais, aspectos emocionais e saúde mental) mostraram associações significativas com TMC ( $\mathrm{p}<0,001)$. As pessoas positivas no rastreio para TMC obtiveram menor média nos escores de qualidade de vida em todos os domínios, quando comparadas com aquelas que pontuaram negativamente (Tabela 3 ).

$\mathrm{Na}$ análise multivariada, demonstrada na Figura 1, a associação entre TMC e qualidade de vida permanece mesmo quando controlada para possíveis fatores de confusão, e ainda com valor de $\mathrm{p}<0,001$ para todos os domínios da SF-36.

\section{Discussão}

Este estudo verificou uma prevalência de 24,5\% de TMC e sua associação com uma pior qualidade de vida quanto a aspectos gerais de saúde em uma população de jovens entre 18 e 24 anos de idade. A prevalência de TMC neste estudo foi similar a outros inquéritos realizados no Brasil que apresentaram de $17 \%$ a $35 \%$ de TMC $2,3,4,5$. As diferenças de resultados entre os estudos podem ser justificadas pelo ponto de corte utilizado.

Além disso, nossos achados quanto às características sociodemográficas também estão de acordo com outros estudos. Um dos fatores que apresentou maior prevalência de TMC foi ser do sexo feminino 23,24,25,26. Um dos achados mais importantes na literatura é que as mulheres têm uma prevalência de ansiedade e de depressão duas a três vezes maior que os homens $3,24,27$. Uma possível justificativa é o fato de que as mulheres têm uma autopercepção de pior saúde do que os homens, sendo assim, expressam com 
Fatores associados aos transtornos mentais comuns (TMC) em jovens de 18 a 24 anos, residentes na cidade de Pelotas, Rio Grande do Sul, Brasil.

\begin{tabular}{|c|c|c|c|c|}
\hline Variáveis & $\begin{array}{c}\text { Descrição da } \\
\text { amostra } \\
\text { n (\%) }\end{array}$ & $\begin{array}{l}\text { Prevalência de TMC } \\
\text { (\%) }\end{array}$ & RP (IC95\%) & Valor de $\mathrm{p}$ \\
\hline \multicolumn{5}{|l|}{ Idade (anos) } \\
\hline $18-20$ & $795(51,4)$ & $186(23,4)$ & 1,00 & 0,306 \\
\hline $21-24$ & $753(48,6)$ & $194(25,8)$ & $1,10(0,92-1,31)$ & \\
\hline \multicolumn{5}{|l|}{ Sexo } \\
\hline Feminino & $880(56,4)$ & $283(32,2)$ & $2,21(1,80-2,71)$ & 0,000 \\
\hline Masculino & $680(43,6)$ & $99(14,6)$ & 1,00 & \\
\hline \multicolumn{5}{|c|}{ Classe socioeconômica } \\
\hline$A+B$ & $583(37,4)$ & $109(18,7)$ & 1,00 & 0,000 \\
\hline C & $751(48,1)$ & $184(24,5)$ & $1,31(1,06-1,62)$ & \\
\hline$D+E$ & $226(14,5)$ & $89(39,4)$ & $2,11(1,67-2,66)$ & \\
\hline \multicolumn{5}{|c|}{ Estuda atualmente } \\
\hline Não & $843(54,0)$ & $231(27,4)$ & $1,30(1,09-1,56)$ & 0,004 \\
\hline $\operatorname{Sim}$ & $717(46,0)$ & $151(21,1)$ & 1,00 & \\
\hline \multicolumn{5}{|c|}{ Trabalha atualmente } \\
\hline Não & $387(40,4)$ & $118(30,5)$ & $1,55(1,24-1,94)$ & 0,000 \\
\hline $\operatorname{Sim}$ & $570(59,6)$ & $112(19,6)$ & 1,00 & \\
\hline \multicolumn{5}{|c|}{ Uso de álcool * } \\
\hline Não & $1.141(73,1)$ & $259(22,7)$ & 1,00 & 0,008 \\
\hline Sim & $419(26,9)$ & $123(29,4)$ & $1,29(1,08-1,55)$ & \\
\hline \multicolumn{5}{|c|}{ Uso de tabaco * } \\
\hline Não & $1.171(75,1)$ & $234(20,0)$ & 1,00 & 0,000 \\
\hline $\operatorname{Sim}$ & $389(24,9)$ & $148(38,0)$ & $1,90(1,60-2,26)$ & \\
\hline \multicolumn{5}{|c|}{ Uso de substâncias ilícitas ** } \\
\hline Não & $1.444(92,6)$ & $320(22,2)$ & 1,00 & 0,000 \\
\hline Sim & $116(7,4)$ & $62(53,4)$ & $2,41(1,98-2,93)$ & \\
\hline
\end{tabular}

* Consumo na última semana;

** Uso nos últimos três meses.

maior facilidade seus sintomas e procuram mais os serviços de saúde 4,28,29.

Pertencer à classe socioeconômica D ou E também se mostrou significativo para o TMC. Segundo Costa \& Ludemir 11, TMC estão relacionados diretamente a fatores socioeconômicos.

Alguns estudos apontam que quanto mais avançada a idade maior a probabilidade de se desenvolver TMC 12,24. O que não se confirmou neste estudo, pois a idade foi a única variável testada que não se mostrou associada à ocorrência de TMC. Uma possível explicação talvez seja a nossa população deste estudo, que se limita a jovens de 18 a 24 anos. Em estudo realizado com jovens de 15 a 18 anos, a idade também não se mostrou associada 2 . Provavelmente, isso seja consequência da pequena margem de idade avaliada em ambos.
Com relação ao uso de substâncias, o uso de álcool esteve associado à presença de TMC como relatado por grande parte da literatura científica 30,31,32,33,34,35,36,37. O tabagismo também mostrou associação significativa para TMC, assim como em um estudo realizado em Pelotas com adolescentes de 15 a 18 anos $(\mathrm{p}<0,001)^{2}$.

A dependência da nicotina pode refletir tanto na hipótese de que fumar pode levar ao desenvolvimento de TMC, quanto de que o transtorno aumenta a probabilidade de tornar-se um fumante regular, uma vez que tabagismo pode estar exercendo a função de automedicação, caso se considere sua ação sobre a atividade dopaminérgica e serotoninérgica 38 .

Assim como nos TMC, menor classificação socioeconômica 7, consumo de substâncias psicoativas 39,40,41,42,43 também são fatores que acar- 
Tabela 2

Variáveis associadas a menores escores entre os domínios de qualidade de vida, em uma amostra de jovens de 18 a 24 anos, residentes na cidade de Pelotas, Rio Grande do Sul, Brasil.

\begin{tabular}{|c|c|c|c|c|c|c|c|c|}
\hline Variáveis independentes & $\begin{array}{l}\text { Capacidade } \\
\text { funcional }\end{array}$ & $\begin{array}{l}\text { Aspectos } \\
\text { físicos }\end{array}$ & Dor & $\begin{array}{c}\text { Estado } \\
\text { geral de } \\
\text { saúde }\end{array}$ & Vitalidade & $\begin{array}{l}\text { Aspectos } \\
\text { sociais }\end{array}$ & $\begin{array}{l}\text { Aspectos } \\
\text { emocionais }\end{array}$ & $\begin{array}{l}\text { Saúde } \\
\text { mental }\end{array}$ \\
\hline Mais velhos (21-24 anos) & 0,002 & 0,298 & 0,392 & 0,763 & 0,403 & 0,857 & 0,407 & 0,996 \\
\hline Sexo feminino & 0,000 & 0,351 & 0,000 & 0,000 & 0,000 & 0,000 & 0,000 & 0,000 \\
\hline Menor classe socioeconômica (D+E) & 0,000 & 0,000 & 0,000 & 0,000 & 0,154 & 0,012 & 0,001 & 0,000 \\
\hline Não estudar & 0,000 & 0,003 & 0,002 & 0,053 & 0,828 & 0,080 & 0,140 & 0,286 \\
\hline Não trabalhar & 0,000 & 0,244 & 0,617 & 0,097 & 0,871 & 0,751 & 0,199 & 0,002 \\
\hline Usar álcool & 0,000 & 0,000 & 0,000 & 0,000 & 0,000 & 0,000 & 0,000 & 0,000 \\
\hline Usar tabaco & 0,285 & 0,018 & 0,000 & 0,000 & 0,003 & 0,000 & 0,000 & 0,002 \\
\hline Usar substâncias ilícitas & 0,000 & 0,000 & 0,000 & 0,000 & 0,000 & 0,000 & 0,000 & 0,000 \\
\hline
\end{tabular}

Tabela 3

Médias e desvio-padrão dos oito domínios de qualidade de vida da Medical Outcomes Survey Short-form General Health Survey (SF-36) entre os desfechos estudados.

\begin{tabular}{|c|c|c|c|c|c|c|}
\hline Domínios da SF-36 & Amostra total & Com TMC & Sem TMC & $\begin{array}{l}\text { Diferença entre } \\
\text { médias (IC95\%) }\end{array}$ & Teste $\mathbf{t}$ & Valor de $p$ \\
\hline Capacidade funcional & $92,2 \pm 12,7$ & $85,4 \pm 17,1$ & $94,4 \pm 10,0$ & $9,0(7,6-10,4)$ & 12,6 & $<0,001$ \\
\hline Aspectos físicos & $85,7 \pm 27,5$ & $69,8 \pm 37,4$ & $90,8 \pm 21,0$ & $21,0(18,0-24,0)$ & 13,7 & $<0,001$ \\
\hline Dor & $75,0 \pm 21,2$ & $61,0 \pm 22,8$ & $79,5 \pm 18,5$ & $18,4(16,1-20,6)$ & 15,9 & $<0,001$ \\
\hline Estado geral de saúde & $77,0 \pm 19,8$ & $63,7 \pm 22,7$ & $81,2 \pm 16,6$ & $17,6(15,4-19,7)$ & 16,3 & $<0,001$ \\
\hline Vitalidade & $60,5 \pm 18,4$ & $43,4 \pm 19,1$ & $66,0 \pm 14,2$ & $22,6(20,8-24,4)$ & 24,7 & $<0,001$ \\
\hline Aspectos sociais & $83,8 \pm 22,2$ & $64,7 \pm 26,9$ & $90,0 \pm 16,2$ & $25,2(23,0-27,5)$ & 22,1 & $<0,001$ \\
\hline Aspectos emocionais & $79,2 \pm 34,0$ & $50,5 \pm 42,1$ & $88,5 \pm 24,5$ & $37,9(34,5-41,4)$ & 21,6 & $<0,001$ \\
\hline Saúde mental & $74,0 \pm 20,4$ & $50,8 \pm 21,2$ & $81,5 \pm 13,3$ & $30,6(28,8-32,4)$ & 33,3 & $<0,001$ \\
\hline
\end{tabular}

TMC: transtornos mentais comuns.

retam prejuízos à qualidade de vida. Quanto ao gênero, nosso estudo mostrou que as mulheres têm maior prevalência de TMC juntamente com escores menores em sete dos oito domínios do SF-36. Estudos apontam uma similaridade quanto aos nossos achados 44,45 .

A qualidade de vida avaliada pelo SF-36 aborda as limitações na vida diária por conte de problemas de saúde, dando uma estimativa subjetiva do estado funcional do indivíduo que, por sua vez, está relacionada à saúde mental. Nos últimos anos há um aumento de pesquisas interessadas em avaliar a qualidade de vida em portadores de transtornos mentais, pelo impacto gerado na vida do indivíduo. Dentre os transtornos associados ao declínio da qualidade de vida está a depressão, por aumentar a sensação de dor e a incapacidade funcional, tornar a adesão ao tratamento mais difícil e diminuir a qualidade das relações sociais $46,47,48$.

No transtorno bipolar os episódios depressivos e os sintomas residuais de depressão, bem como os sintomas de irritabilidade e alterações de sono estão correlacionados à pior qualidade de vida, já que o funcionamento diário do indivíduo está alterado 49 . No transtorno de pânico, fobia social e transtorno de ansiedade generalizada, problemas psicossociais e altos níveis de ansiedade podem comprometer a qualidade de vida 50,51 . As comorbidades entre transtornos de ansiedade e de humor são comuns e apresentam maior prejuízo à qualidade de vida 52,53.

Dessa forma, mesmo que o instrumento SRQ-20 não possibilite a identificação específica do(s) transtorno(s) mental(is) em questão, é provável que a grande maioria dos indivíduos 
Média e desvio-padrão dos domínios da Medical Outcomes Survey Short-form General Health Survey (SF-36) com teste de significância ajustado por regressão linear.

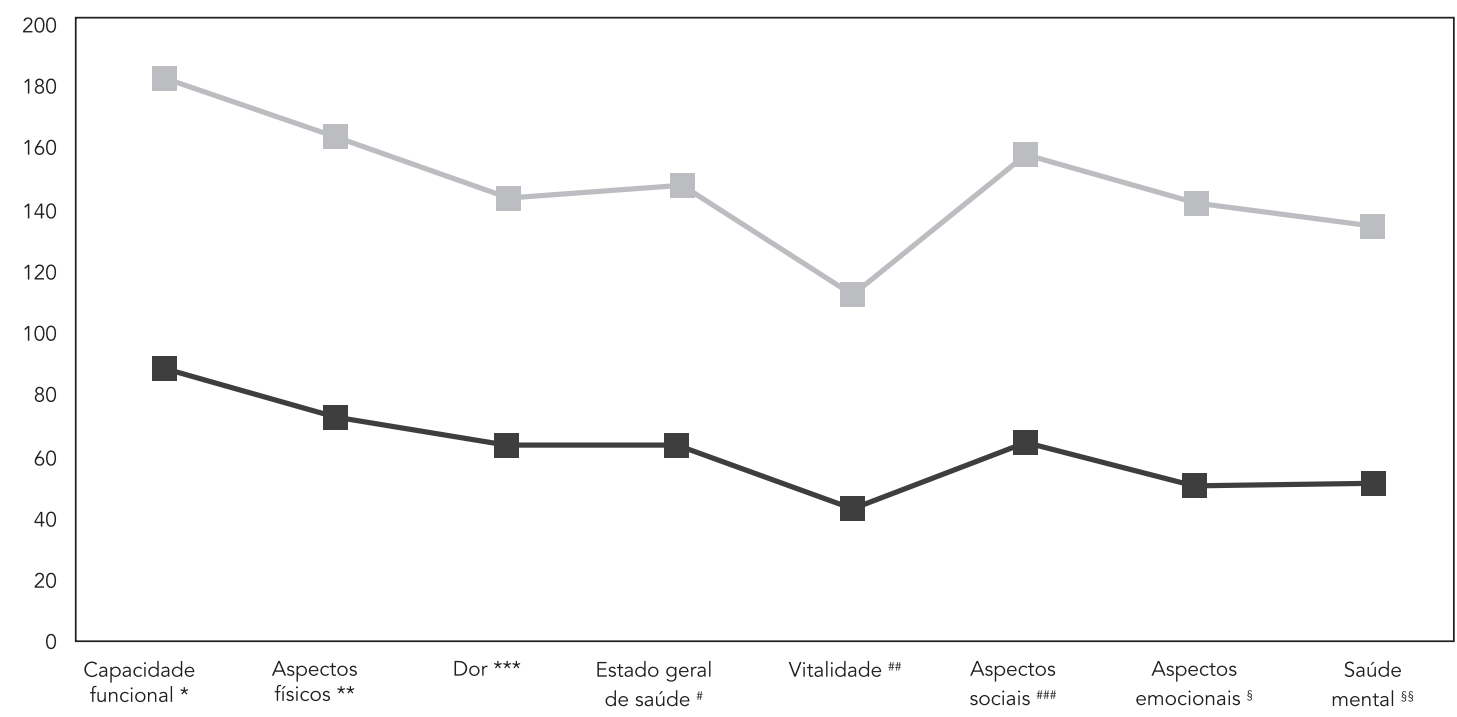

TMC: transtornos mentais comuns.

Nota: valor de $\mathrm{p}<0,05$ em todos os domínios da SF-36, após o ajuste por regressão linear.

* Capacidade funcional ajustada para sexo, classe socioeconômica, estudo, trabalho, uso de álcool e substâncias ilícitas;

** Aspectos físicos ajustados para classe socioeconômica, estudo, uso de álcool, tabaco e substâncias ilícitas;

*** Dor ajustada para sexo, classe socioeconômica, estudo, uso de álcool, tabaco e substâncias ilícitas;

\# Estado geral de saúde ajustado para sexo, classe socioeconômica, estudo, trabalho, uso de álcool, tabaco e substâncias ilícitas;

\#\# Vitalidade ajustada para sexo, classe socioeconômica, uso de álcool, tabaco e substâncias ilícitas;

\#\#\# Aspectos sociais ajustados para sexo, classe socioeconômica, estudo, uso de álcool, tabaco e substâncias ilícitas;

§ Aspectos emocionais ajustados para sexo, classe socioeconômica, estudo, trabalho, uso de álcool, tabaco e substâncias ilícitas;

$\S \S$ Saúde mental ajustada para sexo, classe socioeconômica, trabalho, uso de álcool, tabaco e substâncias ilícitas.

que obteve escore indicativo de TMC apresente algum transtorno de humor e/ou de ansiedade. A utilização de tal instrumento define um conceito abrangente de TMC que pode indicar diferentes transtornos mentais ou condições que devem ser foco de atenção clínica. Ainda assim, o referido instrumento é amplamente utilizado em estudos epidemiológicos, possui aplicação simples e características psicométricas adequadas para o rastreamento de transtornos mentais. O presente estudo apresenta outra limitação que deve ser considerada na interpretação de seus resultados. Ela diz respeito ao seu delineamento transversal que impossibilita a avaliação de qualquer relação causal entre qualidade de vida e a presença de TMC. Porém, é importante ressaltar os aspectos biopsicossociais relacionados aos transtornos mentais, em que o papel da baixa qualidade de vida pode influenciar tanto no surgimento quanto na manutenção de transtornos. Cabe ainda salientar que os achados aqui expostos ganham em credibilidade científica por serem oriundos de uma amostra representativa de jovens.

Os TMC são uma das maiores demandas da atenção em saúde nas classes menos privilegiadas 11,12. Estudo relata que em uma unidade de assistência básica, 50\% dos pacientes apresentavam transtornos mentais 54 . Há prioridade das políticas de saúde para maior atenção ao rastreio de transtornos mentais graves. Sugere-se, assim, que profissionais de saúde na atenção básica atentem para o rastreio dos TMC, sendo ele de alta prevalência na população além de trazer um prejuízo significativo na qualidade de vida da população. Deve-se trabalhar com atenção a prevenção de TMC a fim de proporcionar uma melhor qualidade de vida à população. 


\section{Resumo}

O objetivo foi verificar a prevalência de transtornos mentais comuns (TMC) e sua associação com qualidade de vida em jovens da cidade de Pelotas, Rio Grande do Sul, Brasil. Estudo transversal de base populacional com jovens de 18 a 24 anos. A seleção amostral foi realizada por conglomerados. Para a investigação dos transtornos mentais comuns foi aplicado o Self Report Questionnaire (SRQ-20), enquanto os níveis de qualidade de vida foram mensurados por intermédio da Medical Outcomes Survey Short-form General Health Survey (SF-36). A prevalência de TMC na amostra estudada foi de $24,5 \%$ ( $N=382)$, apresentando-se mais evidente entre as mulheres, entre aqueles que pertenciam à menor classe socioeconômica (D ou E), não estavam estudando, não estavam trabalhando, consumiram álcool e usaram tabaco pelo menos uma vez na última semana e que fizeram uso de alguma substância ilícita nos últimos três meses. Os jovens com TMC obtiveram uma menor média nos escores da SF-36 em todos os domínios de qualidade de vida avaliados. Deve-se investir em medidas preventivas de TMC no intuito de proporcionar uma melhor qualidade de vida à população.

Transtornos Mentais; Saúde Mental; Qualidade de Vida

\section{Colaboradores}

R. A. Silva, L. D. M. Souza e R. T. Pinheiro participaram da elaboração do projeto e coordenação do estudo e aprovaram a revisão final do artigo. T. C. Mondin colaborou na elaboração do projeto, análise dos dados e produção do artigo e aprovou a revisão final do artigo. L. C. Ores contribuiu na elaboração do projeto e coordenação do estudo, produção do artigo e aprovou a revisão final do artigo. K. Jansen participou da elaboração do projeto, análise dos dados e aprovou a revisão final do artigo. C. E. Konradt colaborou na elaboração do projeto e produção do artigo e aprovou a revisão final do artigo.

\section{Agradecimentos}

À Coordenação de Aperfeiçoamento de Pessoal de Nível Superior (CAPES) pelo financiamento.

\section{Referências}

1. Nóbrega FG. Distúrbios da nutrição. Rio de Janeiro: Editora Revinter; 1998.

2. Pinheiro KAT, Horta BL, Pinheiro RT, Horta LL, Terres NG, Silva RA. Common mental disorders in adolescents: a population based cross-sectional study. Rev Bras Psiquiatr 2007; 29:241-5.

3. Ludermir BA, Melo Filho DA. Condições de vida e estrutura ocupacional associadas a transtornos mentais comuns. Rev Saúde Pública 2002; 36: 213-21.

4. Lima MCP, Menezes PR, Carandina L, Cesar CLG, Barros MBA, Goldbaum M. Transtornos mentais comuns e uso de psicofármacos: impacto das condições socioeconômicas. Rev Saúde Pública 2008; 42:717-23.

5. Leon-Marín L, Oliveira HB, Barros MBA, Dalgalarrondo P, Botega NJ. Social inequality and common mental disorders. Rev Bras Psiquiatr 2007; 29:250-3.
6. Patel V, Araya N, Chowdhary N, King M, Kirkwood B, Nayak G, et al. Detecting common mental disorders in primary care in India: a comparison of five screening questionnaires. Psychol Med 2008; 38:221-8.

7. Puertas G, Rios C, Valle H. Prevalencia de trastornos mentales comunes en barrios marginales urbanos con población desplezada en Colombia. Rev Panam Salud Pública 2006; 20:324-30.

8. Jacob KS, Bhugrad D, Lloyd KR, Mann AH. Common mental disorders, explanatory models and consultation behaviour among Indian women living in the UK. J R Soc Med 1998; 91:66-71.

9. Mumford DB, Nazir M, Jilanic FU, Baig IY. Stress and psychiatric disorder in the Hindu Kush: a community survey of mountain villages in Chitral, Pakistan. Br J Psychiatry 1996; 168:299-307. 
10. Goldberg D, Huxley P. Common mental disorders: a bio-social model. London: Tavistock; 1992.

11. Costa AG, Ludermir AB. Transtornos mentais comuns e apoio social: estudo em comunidade rural da Zona da Mata de Pernambuco, Brasil. Cad Saúde Pública 2005; 21:73-9.

12. Lima MS, Béria JU, Tomasi E, Conceição AT, Mari JJ. Stressful life events and minor psychiatric disorders: an estimate of the population attributable fraction in a Brazilian community-based study. Int J Psychiatry Med 1996; 26:211-22.

13. Bond M. The pursuit of happiness. New Sci 2003; 179:40-4.

14. Rubin HC, Rapaport MH, Levine B, Gladsjo JK, Rabin A, Auerbach M, et al. Quality of well being in panic disorder: the assessment of psychiatric and general disability. J Affect Disord 2000; 57:217-21.

15. Schonfeld WH, Verboncoeur CJ, Fifer SK, Lipschutz RC, Lubeck DP, Buesching DP. The functioning and well-being of patients with unrecognized anxiety disorders and major depressive disorder. J Affect Disord 1997; 43:105-19.

16. The World Health Organization Quality of Life assessment (WHOQOL): position paper from the World Health Organization. Soc Sci Med 1995; 41:1403-9.

17. Basu D. Quality of life issues in mental health care: past, present and future. German Journal of Psychiatry 2004; 7:35-43.

18. Fonseca MLG, Guimarães MBL, Vasconcelos EM. Sofrimento difuso e transtornos mentais comuns: uma revisão bibliográfica. Rev APS 2008; 11 : 285-94.

19. Mari JJ, Williams P. A validity study of a psychiatric screening questionnaire (SRQ-20) in primary care in the city of São Paulo. Br J Psychiatry 1986; 148:23-6.

20. Gonçalves DM, Stein AT, Kapczinski F. Avaliação de desempenho do Self-Reporting Questionnaire como instrumento de rastreamento psiquiátrico: um estudo comparativo com o Structured Clinical Interview for DSM-IV-TR. Cad Saúde Pública 2008; 24:380-90.

21. Ciconelli RM, Ferraz MB, Santos W, Meinão I. Tradução para a língua portuguesa e validação do questionário genérico de avaliação de qualidade de vida SF-36 (Brasil SF-36). Rev Bras Reumatol 1999; 39:143-50.

22. Victora CG, Huttly SR, Fuchs SC, Olinto MT. The role of conceptual frameworks in epidemiological analysis: a hierarchical approach. Int J Epidemiol 1997; 26:224-7.

23. Lopes CS, Faerstein E, Chor D. Eventos de vida produtores de estresse e transtornos mentais comuns: resultados do Estudo Pró-Saúde. Cad Saúde Pública 2003; 19:1713-20.

24. Patel V, Araya R, Lima M, Ludermir A, Todd C. Women, poverty and common mental disorders in four restructuring societies. Soc Sci Med 1999; 49:1461-71.

25. Lima MCP. Transtornos mentais comuns e uso de álcool na população urbana de Botucatu [Tese de Doutorado]. São Paulo: Universidade de São Paulo; 2005 .

26. Silva MC, Fassa AG, Kriebel D. Minor psychiatric disorders among Brazilian ragpickers: a cross-sectional study. Environ Health 2006; 5:17.
27. Rojas G, Araya R, Lewis G. Comparing sex inequalities in common affective disorders across countries: Great Britain and Chile. Soc Sci Med 2005; 60:1693-703.

28. Jenkins R. Sex difference in minor psychiatric morbidity. Cambridge: Cambridge University Press; 1985.

29. Schraiber LB, D'Oliveira AFPL, Falcão MTC, Figueiredo WS. Violência dói e não é direito: a violência contra a mulher, saúde e os direitos humanos. São Paulo: Editora UNESP; 2005.

30. Gentil AF, Mathis MA, Torresan RC, Diniz JB, Alvarenga P, Rosário MC, et al. Alcohol use disorders in patients with obsessive-compulsive disorder: the importance of appropriate dual-diagnosis. Drug Alcohol Depend 2009; 100:173-7.

31. Cardoso BM, Kauer Sant'Anna M, Dias VV, Andreazza AC, Ceresér KM, Kapczinski F. The impact of co-morbid alcohol use disorder in bipolar patients. Alcohol 2008; 42:451-7.

32. Fidalgo TM, Silveira ED, Silveira DX. Psychiatric comorbidity related to alcohol use among adolescents. Am J Drug Alcohol Abuse 2008; 34:83-9.

33. Terra MB, Barros HM, Stein AT, Figueira I, Athayde LD, Spanemberg L, et al. Does co-occurring social phobia interfere with alcoholism treatment adherence and relapse? J Subst Abuse Treat 2006; 31:403-9.

34. Terra MB, Barros HM, Stein AT, Figueira I, Jorge MR, Palermo LH, et al. Social anxiety disorder in 300 patients hospitalized for alcoholism in Brazil: high prevalence and undertreatment. Compr Psychiat 2006; 47:463-7.

35. Lima AF, Pechansky F, Fleck MP, De Boni R. Association between psychiatric symptoms and severity of alcohol dependence in a sample of brazilian men. J Nerv Ment Dis 2005; 193:126-30.

36. Alves H, Kessler F, Ratto LR. Comorbidade: uso de álcool e outros transtornos psiquiátricos. Rev Bras Psiquiatr 2004; 26 Suppl 1:S51-3.

37. Laranjeira R. Álcool: da saúde pública à comorbidade psiquiátrica. Rev Bras Psiquiatr 2004; 26 Suppl 1:S1-2.

38. Vieyra-Reyes P, Venebra-Muñoz A, Rivas-Santiago B, García-García F. Acción de la nicotina como antidepresivo y regulador del sueño en sujetos deprimidos. Rev Neurol 2009; 49:661-7.

39. Schmitz N, Kruse J, Kugler J. Disabilities, quality of life, and mental disorders associated with smoking and nicotine dependence. Am J Psychiatry 2003; 160:1670-6.

40. Castro MG, Oliveira MS, Moraes JFD, Miguel AC, Araujo RB. Qualidade de vida e gravidade da dependência de tabaco. Rev Psiquiatr Clín (São Paulo) 2007; 34:61-7.

41. Chen CY, Storr CL. Alcohol use and health-related quality of life among youth in Taiwan. J Adolesc Health 2006; 39:752.

42. Lima AFBS. Qualidade de vida em pacientes do sexo masculino dependentes de álcool [Dissertação de Mestrado]. Porto Alegre: Universidade Federal do Rio Grande do Sul; 2002.

43. Falck RS, Wang J, Carlson RG, Siegal HA. Crackcocaine use and health status as defined by the SF36. Addict Behav 2000; 25:579-84. 
44. Kunkel N, Oliveira WF, Peres MA. Excesso de peso e qualidade de vida relacionada à saúde em adolescentes de Florianópolis, SC. Rev Saúde Pública 2009; 42: 226-35.

45. Lopes GB, Martins MTS, Matos CM, Amorim JL, Leite EB, Miranda EA, et al. Comparações de medidas de qualidade de vida entre mulheres e homens em hemodiálise. Rev Assoc Med Bras 2007; 53:506-9.

46. Berber JSS, Kupek E, Berber SC. Prevalência de depressão e sua relação com a qualidade de vida em pacientes com síndrome da fibromialgia. Rev Bras Reumatol 2005; 45:47-54.

47. Santos AMB, Assumpção A, Matsutani LA, Pereira CAB, Lage LV, Marques AP. Depressão e qualidade de vida em pacientes com fibromialgia. Rev Bras Fisioter 2006; 10:317-24.

48. Couto ER, Couto E, Vian B, Gregório Z, Nomura ML, Zaccaria R, et al. Quality of life, depression and anxiety among pregnant women with previous adverse pregnancy outcomes. São Paulo Med J 2009; 127:185-9.

49. Gazalle FK. Fatores associados à qualidade de vida em pacientes com transtorno bipolar [Dissertação de Mestrado]. Porto Alegre: Universidade Federal do Rio Grande do Sul; 2008.
50. Melo-Neto VL, Valença AM, Nascimento I, Lopes FL, Nardi AE. Quality of life assessment by WHOQOL-BREF in panic disorder patients during treatment. Rev Psiquiatr Clín (São Paulo) 2008; 35:49-54.

51. Barrera TL, Norton PJ. Quality of life impairment in generalized anxiety disorder, social phobia, and panic disorder. J Anxiety Disord 2009; 23:1086-90.

52. Wittchen HU, Carter RM, Pfister H, Montgomery SA, Kessler RC. Disabilities and quality of life in pure and comorbid generalized anxiety disorder and major depression in a national survey. Int Clin Psychopharmacol 2000; 15:319-28.

53. Norberg MM, Diefenbach GJ, Tolin DF. Quality of life and anxiety and depressive disorder comorbidity. J Anxiety Disord 2008; 22:1516-22.

54. Gonçalves DM, Kapczinski F. Prevalência de transtornos mentais em indivíduos de uma unidade de referência para Programa Saúde da Família em Santa Cruz do Sul, Rio Grande do Sul, Brasil. Cad Saúde Pública 2008; 24:2043-53.

Recebido em 22/Jun/2010

Versão final reapresentada em 09/Nov/2010 Aprovado em 23/Dez/2010 